\title{
Laboreal
}

Volume $6 \mathrm{~N}^{\circ} 2$ | 2010

Varia

\section{Para una contextualización de la prevención en seguridad y salud ocupacionales en el sector de la construcción : contribuciones de la formación de coordinadores de seguridad y salud}

Para uma contextualização da prevenção em segurança e saúde ocupacional no sector da construção : contributos da formação de coordenadores de segurança e saúde

Promotion de la contextualisation de la prévention en sécurité et santé au travail dans le bâtiment : contributions de la formation en coordination en sécurité et santé

Promoting work safety and health prevention contextualization in building sector : contributions of the safety and health coordinators' training

\section{Camilo Valverde}

\section{OpenEdition}

Journals

Edición electrónica

URL: http://journals.openedition.org/laboreal/8856

DOI: 10.4000/laboreal.8856

ISSN: 1646-5237

Editor

Universidade do Porto

Referencia electrónica

Camilo Valverde, «Para una contextualización de la prevención en seguridad y salud ocupacionales en el sector de la construcción : contribuciones de la formación de coordinadores de seguridad y salud », Laboreal [En línea], Volume 6 No2 | 2010, Publicado el 01 diciembre 2010, consultado el 24 septiembre 2020. URL : http://journals.openedition.org/laboreal/8856 ; DOI : https://doi.org/10.4000/ laboreal.8856

Este documento fue generado automáticamente el 24 septiembre 2020.

Laboreal está licenciado com uma Licença Creative Commons - Atribuição-NãoComercial 4.0 Internacional. 


\section{Para una contextualización de la prevención en seguridad y salud ocupacionales en el sector de la construcción : contribuciones de la formación de coordinadores de seguridad y salud}

Para uma contextualização da prevenção em segurança e saúde ocupacional no sector da construção : contributos da formação de coordenadores de segurança e saúde

Promotion de la contextualisation de la prévention en sécurité et santé au travail dans le bâtiment : contributions de la formation en coordination en sécurité et santé

Promoting work safety and health prevention contextualization in building sector : contributions of the safety and health coordinators' training

Camilo Valverde

\section{REFERENCIA}

Valverde, C. (2010). Para uma contextualização da prevenção em segurança e saúde ocupacional no sector da construção : contributos da formação de coordenadores de segurança e saúde. Tese de Doutoramento, Faculdade de Psicologia e de Ciências de Educação da Universidade do Porto, Porto. 


\section{NOTA DEL EDITOR}

Manuscrito recibido en : noviembre/2010

Aceptado tras peritaje en : diciembre/2010

\section{Contexto y problemática}

1 Este trabajo fue realizado en Portugal en el contexto del sector de la construcción civil y las obras públicas. Esta era una industria marcada por la elevada siniestralidad (la tasa de frecuencia de accidentes y el porcentaje de accidentes mortales tenía valores encima de la media comparados con otras industrias y servicios [1]), por inestabilidad organizativa (con uso masivo de la subcontratación) y laboral (con predominio de relaciones de trabajo poco reglamentadas y precarias), operando en un contexto económico y social de gran competitividad y elevado desempleo en el que ocurrían cambios tecnológicos relevantes. Los modelos que existían sobre gestión de la prevención en la seguridad y salud en el trabajo, basados en la tradicional prescripción de procedimientos de tipo regulativo y técnico, y en el control y la verificación de la conformidad, encontraban dificultad para enfrentarse a la variabilidad, la incertidumbre, la complejidad y las singularidades que caracterizaban este sector.

2 En este marco apareció, como una alternativa adaptada, el surgimiento en la industria de la construcción de la primera Directiva Sectorial sobre higiene, seguridad y salud en el trabajo: la Directiva Astilleros (92/57/CEE) [2] sobre disposiciones mínimas de seguridad y salud en el trabajo a cumplir en los astilleros temporales o móviles, que previó la creación de una función específica - la del Coordinador en seguridad y salud en la construcción, para intervenir en todas las etapas del proceso de construcción: desde su concepción hasta la demolición - con el fin de desarrollar la gestión de la prevención en un sector económico en el que las formas predominantes de regulación y gestión de la prevención se han mostraban ineficaces.

3 Sin embargo, si la forma de concebir, implementar y regular las intervenciones preventivas por parte de este nuevo protagonista se fundamentan en una tendencia reduccionista, con un abordaje de naturaleza técnico-reglamentaria ; será una decisión poco consecuente, sustentada en una lógica incongruente: enfrentarse a problemas específicos mediante el uso de conceptos y herramientas de análisis y de acción de carácter general, que resultaban claramente insuficientes frente a la diversidad de situaciones que se observaban en el terreno. Es en este contexto que consideramos pertinente nuestra propuesta en términos de promover la acción preventiva de los Coordinadores de seguridad y salud, a partir del análisis y la transformación de los procesos de trabajo, teniendo en cuenta sus condiciones concretas en las dinámicas específicas de los astilleros.

4 Para ello, seleccionamos como objeto de estudio el análisis de la formación de estos nuevos actores, como posible vía para la trans-formación de sus representaciones y la apertura de oportunidades para otras lógicas de gestión de la prevención. En particular, si esta formación se basa en un enfoque reflexivo de las prácticas y de las concepciones de la prevención, orientada a la construcción de un colectivo profesional de preventores mejor instrumentados para hacer frente a las discontinuidades que se han venido imponiendo en los sistemas productivos, con potencial para generar nuevos 
riesgos profesionales, para los que se hace necesario repensar diferentes formas de evaluación y de prevención.

\section{Marco científico-metodológico}

Adoptamos el enfoque teórico-metodológico denominado "paradigma de la formación de los actores" incluido en la tradición científica de la Psicología del Trabajo y la Ergonomía de la actividad, que confiere un estatuto privilegiado al análisis de las actividades laborales como una dimensión estructurada de los procesos de investigación y de intervención, equiparando las relaciones entre el análisis del trabajo, la gestión de la prevención en seguridad y salud del trabajo, y la formación de los factores que actúan en este campo (Lacomblez, 2001; Lacomblez \& Teiger, 2006; Teiger,, 2002 ; Lacomblez \& Teiger, 2001; Teiger, \& Lacomblez , 2005 ; Lacomblez \& Teiger, 2006). Este es un proyecto científico que asume como finalidad la adaptación del trabajo al Hombre y busca valorizar una gestión alternativa de la seguridad y el bienestar de los trabajadores atendiendo a su desarrollo individual y colectivo. Para comprender y transformar las situaciones de trabajo, esta perspectiva establece la distinción entre el trabajo prescrito (la tarea) y el trabajo real (la actividad). Además, se interroga de manera frontal las diferencias de estatus, que normalmente se atribuyen a los conocimientos de los actores, considerados como especialistas y no especialistas, valorizando la forma de compartir, de modo no jerárquico, sus distintas experiencias. Sostiene, también, que las concepciones y las prácticas de prevención se llevan a cabo y se estructuran siempre a partir del estudio multidisciplinario de los riesgos en el contexto laboral en que ocurren, en una dinámica participativa que moviliza los diversos actores involucrados.

6 El trabajo de campo adoptó un punto de vista metodológico de carácter cualitativo, llevando a cabo una lógica de estudio de caso en el terreno, mediante la realización de dos estudios: la gestión de la prevención en la construcción del Puente Infante D. Henrique en Oporto, y un programa de capacitación en Coordinación de Seguridad y Salud en la construcción, realizado por los Ingenieros de la Región Norte. El primer estudio, que muestra algunas de las características específicas del sector, contó con el seguimiento de la ejecución, teniendo para ello acceso a dispositivos metodológicos como las observaciones en el terreno, los análisis de documentos y las entrevistas con los especialistas en prevención; con el fin de identificar los elementos que los factores involucrados en la ejecución de la obra tuvieron en cuenta, para caracterizar las concepciones y las prácticas subyacentes a las intervenciones que ocurrieron en materia de seguridad y salud. El análisis de contenido de las entrevistas con 14 protagonistas en prevención (agrupados en los siguientes grupos profesionales : 3 operadores, 3 jefes de base, 3 altos directivos, 3 técnicos/administrativos y 2 técnicos de seguridad) se realizó mediante un instrumento informático especializado denominado TROPES, obteniendo categorías que fueron interpretadas con la ayuda del análisis de componentes principales o CATPCA - Categorical Principal Component Analysis (Meulman \& Heiser, 2005). En el segundo estudio, describimos los contenidos y los procesos de un programa de formación para Coordinadores de seguridad y salud, promovido por una entidad de referencia en esta área, para, de acuerdo con nuestro marco teórico, sugerir cambios en la formación de estos profesionales. 


\section{Análisis y discusión de los resultados}

7 El estudio de caso sobre el programa de prevención implementado en la construcción del Puente Infante D. Henrique y el análisis de las representaciones de los especialistas en prevención involucrados en su gestión, mostró una gran diversidad en las formas de concebir y de realizar la gestión de la prevención. De hecho, los resultados mostraron claramente que en los actores que protagonizaron la gestión de la prevención en la construcción del puente estudiado, hubo una clara distinción entre el mundo [ $\left.{ }^{3}\right]$ (véase el Béguin, 2005) de la prevención de los operadores y supervisores próximos y el mundo de la prevención de los técnicos de seguridad y los altos directivos. Al parecer, estamos ante dos mundos que no se encuentran y que parecen traducir formas muy diferentes de concebir, y eventualmente de realizar, la prevención de riesgos profesionales y la promoción del bienestar de los trabajadores involucrados en la gestión de la seguridad en el astillero.

8 Esta conclusión establece importantes implicaciones para la intervención y, por lo tanto, para la formación de los Coordinadores de seguridad y salud que, teniendo la misión de garantizar la seguridad y promover la salud en el sector de la construcción civil y las obras públicas, tienen que estar preparados para comprender estas situaciones y para promover acciones reflexivas, de compromiso, comunicación e intercambio de experiencias que faciliten formas comunes de ver : una construcción de mundos compartidos (Béguin, 2005).

9 En el estudio del Programa de Formación de Coordinadores de seguridad y salud en la construcción, a través del análisis de los temas previstos y la observación participante, resultante de la frecuencia de las sesiones desarrolladas, se constató que los contenidos estaban centrados fundamentalmente en torno a cuestiones de naturaleza legal y de naturaleza técnica. Haciéndose especial hincapié en la legislación, la regulación y la limitación de la forma de actuar de carácter técnico y administrativo, operando con lógicas de tipo normativas, de control, conformidad, comprobación del cumplimiento y de prescripción. También consideramos que los enfoques preventivos llevados a cabo, tendían a estar claramente fundamentados en procedimientos y en normas de aplicación general, supuestamente consideradas como universales, sin tener en cuenta las condiciones concretas en las que se realizaban las actividades laborales. Por regla general, no se tenía en cuenta la gestión de la variabilidad, lo aleatorio, lo imprevisto y lo incierto, muy común en la vida cotidiana de los astilleros de la construcción.

10 Emergió, así, la importancia de preguntarse sobre la posibilidad de este tipo de entrenamiento, claramente tributaria del paradigma que hemos venido a llamar de técnico-normativo, para contribuir a que la intervención en el sitio sea promotora de un mundo de la prevención, traducido por una localización de los aspectos relacionados con la verificación de la conformidad con las normas y los procedimientos establecidos.

\section{Conclusiones y posibles vías de reflexión.}

11 Para promover la construcción de mundos compartidos, el paradigma de formación de los actores preconiza el anclaje de la prevención en las condiciones concretas de realización de la actividad laboral en el terreno, y propone el intercambio/encuentro no jerárquico de conocimientos/experiencias, sustentando la creación de comunidades 
científicas ampliadas y la promoción de interfaces entre los actores. Este enfoque de formación se revela bien adaptado para cotejar la capacitación en Coordinación de seguridad y salud y para contribuir a la contextualización de la prevención en el sector de la construcción.

Por consiguiente, podríamos concluir que formar a los actores de la prevención requiere que se elija, como unidad de análisis, no al individuo, sino a la red de interacciones involucradas en la obra. Esta red de intercambio es el resultado de la conjugación/alineamiento de los factores con puntos de vista diversos, con formas de ver e identidades diferentes, que son movilizados para la realización de la obra. Para que esta ejecución sea más efectiva, es necesario que los actores se articulen y que converjan en los posicionamientos, en las lógicas y los puntos de vista diferentes que, sin que se absorban/reduzcan unos a otros, contribuyan a la concretización de las actividades conjuntas necesarias para la obra, integrando la seguridad y el bienestar de forma sostenible.

Esta propuesta requiere la creación de puntos de contacto en los astilleros para conocer y reconocer los diferentes mundos, y para subsecuentemente, promover la construcción de "mundos compartidos". Se abren, de esta forma, nuevas posibilidades para la sinergia entre los medios de prevención y la Psicología del Trabajo en todo el paradigma de la formación de los actores, cuyos ejes estructurales son, precisamente, el anclaje en las situaciones de trabajo concretas y la valorización de los intercambios entre los diferentes registros de conocimiento (Lacomblez \& Teiger, 2001). Por lo tanto, admitir que el paradigma de la formación de actores, propuesto por la Psicología del Trabajo y por la Ergonomía de la Actividad, aparentemente, revela una particular adecuación en la perspectiva de la formación de Coordinadores de seguridad y la salud, entendidos en su papel preventivo como gestores de interfaces del sector.

De hecho, la profesión/actividad de los Coordinadores de la seguridad y la salud, y de los actores de la prevención, en general, puede reestructurarse/reconstruirse, de forma ventajosa, en un proceso de formación sobre análisis ergonómico del trabajo. Este proceso de capacitación tiene el potencial de inducir modulaciones distintas en el posicionamiento y el conocimiento de los preventores: les permite ampliar, profundizar y, eventualmente, volver a configurar las relaciones que se van a establecer entre la formación, el trabajo y la prevención. En estos procesos de desarrollo es posible que los actores de la prevención se enfrenten con nuevas contradicciones del registro "intención paradójica" entre las "conformidades" con los reglamentos/procedimientos, que rara vez son coherente con las limitaciones reales de la actividad en un contexto específico $\mathrm{y}$, en algunas situaciones, las conformidades probablemente se perciban como barreras para la prevención. Esta situación ilustra la necesidad de tener que considerar las condiciones del contexto (sociales, organizacionales, administrativas...) que son necesarias para garantizar la sostenibilidad de este tipo de intervenciones (Lacomblez y Teige, 2001). 


\section{BIBLIOGRAFÍA}

Alves Dias, L. (2005). Segurança e saúde no trabalho da construção na União Europeia. Segurança, 168, Suplemento Especial, Novembro/Dezembro, 7-22.

Béguin, P. (2005). Concevoir pour les genèses professionnelles. In P. Rabardel \& P. Pastré (dir.), Modèles du sujet pour la conception (pp. 31-52). Toulouse : Octarès Éditions.

IGT (2004). Campanha europeia da construção 2003. [Em linha] Disponível em http:// www.igt.gov.pt/DownLoads/content/REL_FINAL_CAMP_EUR_CONST2003.pdf [Consultado em 11/06/2005].

IGT (2005). Coordenação da segurança e saúde do trabalho nos empreendimentos da construção. [Em linha] Disponível em http://www.igt.gov.pt/DownLoads/content/ Coordenacao_trabalho_empreendimentos_construcao.pdf [Consultado em 13/02/2006].

Lacomblez, M. (2001). Analyse du travail et élaboration des programmes de formation professionnelle. Relations Industrielles, 56, 3, 543-578.

Lacomblez, M., \& Teiger, C. (2006). Ergonomia, formações e transformações. In P. Falzon (Ed.), Ergonomia. (pp. 587-602). S. Paulo: Edgard Blücher.

Meulman, J., \& Heiser, W. (2005). SPSS Categories. Chicago: SPSS Inc.

Teiger, C. (2002). Origines et évolution de la formation à la prévention de risques " gestes et postures » en France. Relations Industrielles, 57,3, 431-462.

Teiger, C., \& Lacomblez, M. (2001). Introduction des sessions du Séminaire “(Se) former pour transformer le travail », Paris : Conservatoire National des Arts et Métiers, Mai.

Teiger, C., \& Lacomblez, M. (2005). L'ergonomie et la trans-formation du travail et/ou des personnes : permanences et évolutions (1). Education Permanente, 165 (4), 9-28.

Teiger, C., \& Lacomblez, M. (2006). L'ergonomie et la trans-formation du travail et/ou des personnes : permanences et évolutions (2). Education Permanente, 166 (1), 9-28.

\section{NOTAS}

1. De acuerdo con datos de Alves Dias (2005) y de la Inspección General del Trabajo (IGT, 2004 e 2005).

2. La transposición de la Directiva Astilleros para el ordenamiento jurídico de nuestro país ocurrió a través del Decreto-Ley 155/95, que fue posteriormente objeto de esclarecimiento y revisión por el Decreto-Ley 273/2003 de 29 de Octubre, actualmente en vigor.

3. Béguin (2005) propone la siguiente operacionalización para el concepto de mundo: "podemos definir el mundo como una apropiación, por el sujeto, de ciertas propiedades de lo real, en el marco de su actividad histórica y socialmente situada" (p. 33, traducción libre). El mismo autor, considera que "un mundo consiste en los paños de fondo conceptuales, axiológicos y prácticos que forman el sistema con los objetos de acción" (ídem, p.35, traducción libre). 


\section{AUTOR}

\section{CAMILO VALVERDE}

Faculdade de Economia e Gestão da Universidade Católica Portuguesa, Rua Diogo Botelho, 1327, 4169-005 Porto. Centro de Psicologia da Universidade do Porto, Rua do Dr. Manuel Pereira da Silva, 4200-392 Porto

cvalverde@porto.ucp.pt 